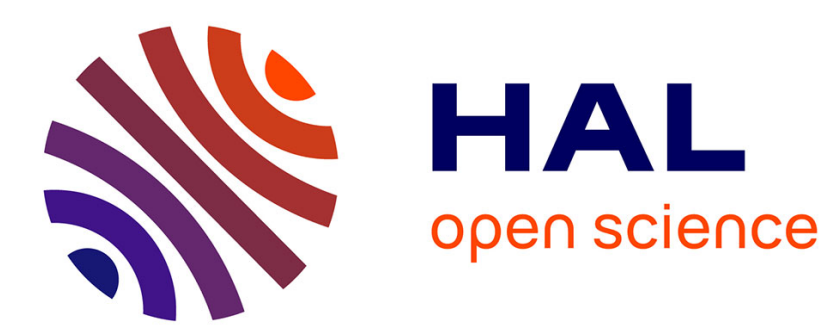

\title{
On Bose occupancy problem with randomized energy levels
}

\author{
Thierry Huillet, Flora Koukiou
}

\section{To cite this version:}

Thierry Huillet, Flora Koukiou. On Bose occupancy problem with randomized energy levels. Journal of Physics A: Mathematical and Theoretical, 2008, Volume 41 (Number 6), pp.065002. hal-00199939

\section{HAL Id: hal-00199939 \\ https://hal.science/hal-00199939}

Submitted on 20 Dec 2007

HAL is a multi-disciplinary open access archive for the deposit and dissemination of scientific research documents, whether they are published or not. The documents may come from teaching and research institutions in France or abroad, or from public or private research centers.
L'archive ouverte pluridisciplinaire HAL, est destinée au dépôt et à la diffusion de documents scientifiques de niveau recherche, publiés ou non, émanant des établissements d'enseignement et de recherche français ou étrangers, des laboratoires publics ou privés. 


\title{
On Bose occupancy problem with randomized energy levels
}

\author{
T. Huillet and F. Koukiou \\ Laboratoire de Physique Théorique et Modélisation, \\ CNRS-UMR 8089 et Université de Cergy-Pontoise, \\ 2, Avenue Adolphe Chauvin, 95302, Cergy-Pontoise, France
}

September 11, 2007

\begin{abstract}
Assuming energy states to be an independent and identically distributed positive sequence, a randomized version of the classical Bose occupancy problem is investigated.
\end{abstract}

Keywords: Disordered systems (Theory), Bose Einstein condensation (Theory), Energy landscapes (Theory), Extreme value problems.

\section{Introduction}

Classical Bose occupancy problem consists in assigning indistinguishable particles on a prescribed deterministic energy states sequence. The purpose of this work is to initiate the study of the following related problem: Assuming now energy states to constitute an independent and identically distributed sequence of positive random variables, we wish to investigate the corresponding randomized version of classical Bose occupancy problem. This simple model does not seem to have received much attention in the past, to the best of the authors knowledge. Under such conditions, using a grand canonical approach, a Bose-Einstein like condensation phenomenon is shown to take place provided the probability mass at zero of energies is small enough (else, probability mass of energies is not "too strongly concentrated" at zero); we shall describe this phase transition in some details and give some illustrative examples. The origin of this phenomenon seems to result from the conjunction of two main effects: The randomness of the energy levels (the system is disordered) and the indistinguishability of particles to be allocated. When there is condensation, we shall discuss the problems of understanding the ground state occupancy at critical density, together with the number of distinct occupied states. Some limit laws are obtained in some cases. 
Also, some aspects of ground state occupancy in the condensed phase will be investigated. Finally, using a description of occupancies of the ranked energy states (the sampling formula), we shall derive the grand-canonical statistics of states with a prescribed amount of particles. This statistical aspect concerns the fluid phase only. We report on a Poisson factorization of this distribution in a specific low temperature, low density limit.

\section{Randomized Bose samples}

\subsection{The model and some of its statistical features}

Let $\mathbf{E}_{n}:=\left(E_{1}, . ., E_{n}\right)$ be a system of independent and identically distributed (iid) positive energies whose law will be specified later on (at this step, we shall however further assume that $E_{1}$ has a density $f_{1}$ with support $\subseteq \mathbb{R}_{+}$and that the largest value from $\mathbf{E}_{n}$ goes to $\infty$ almost surely). Let $\beta>0$ be the inverse of temperature. We shall presently study the randomized energy version of the classical allocation model of statistical mechanics where energy levels are originally chosen deterministically. For some related aspects of the randomized problem, see Johnson and Kotz [12]. The problem under study can now be formulated as follows.

Let there be $k$ indistinguishable particles to place on the random energy levels (or states) $\mathbf{E}_{n}$. Conditionally on $\mathbf{E}_{n}$ (for quenched disorder), let $K_{n, k}(m)$ denote the occupancy of energy level $m$ with Bose equilibrium collective law given by

$$
\mathbb{P}_{\mathbf{E}_{n}}\left(K_{n, k}(m)=k_{m} ; m=1, . ., n\right)=\frac{1}{Z_{k, \mathbf{E}_{n}}(\beta)} \prod_{m=1}^{n} e^{-\beta E_{m} k_{m}} .
$$

With $\left[z^{k}\right] f(z)$ the coefficient of $z^{k}$ in the power-series expansion of $f(z)$ the normalizing term reads

$$
Z_{k, \mathbf{E}_{n}}(\beta)=\sum_{\substack{k_{1}^{\prime}+. .+k_{n}^{\prime}=k \\ k_{1}^{\prime}, \ldots, k_{n}^{\prime} \geq 0}} \prod_{m=1}^{n} e^{-\beta E_{m} k_{m}^{\prime}}=\left[z^{k}\right] \prod_{m=1}^{n}\left(1-z e^{-\beta E_{m}}\right)^{-1}
$$

The distribution defined by Eqs. (2.1, 2.2) favors configurations with minimal (interaction free) energy $H_{n, k}\left(\mathbf{E}_{n}\right):=\sum_{m=1}^{n} E_{m} k_{m}$. Given $\mathbf{E}_{n}$, it is the one maximizing entropy under the assumption that the average energy is fixed and $\beta$ stands for the conjugate parameter. Here and throughout, the subscript $\mathbf{E}_{n}$ in $\mathbb{P}_{\mathbf{E}_{n}}$ (or $\mathbb{E}_{\mathbf{E}_{n}}$ ) will denote conditional probability (or expectation) given $\mathbf{E}_{n}$.

\section{Remarks:}


(i) In Eq. (2.1), $k_{m} \in \mathbb{N}_{0}:=\{0,1,2, .\},. m=1, . ., n$ with no restriction but $k_{1}+. .+k_{n}=k$. Imposing the additional condition that $k_{m} \in\{0,1\}, m=1, . ., n$ (the Pauli exclusion principle), would lead to a Fermi-Dirac occupancy problem which we shall not further develop specifically.

(ii) The classical Bose occupancy problem ([3]) can be obtained by considering an ordered deterministic energy sequence $\boldsymbol{\epsilon}_{(n)}:=\left(\epsilon_{(1)}, . ., \epsilon_{(n)}\right)$ where $\epsilon_{(1)}<. .<\epsilon_{(n)}$, instead of $\mathbf{E}_{n}$ used in $(2.1,2.2)$. Typically, $\epsilon_{(m)}=m^{\alpha}$ for some $\alpha>1$; see [4], for example. We therefore wish to investigate the role of randomization of the energy sequence, requiring an additional averaging over disorder. $\diamond$

Indeed, given there are $k$ particles, averaging Eq. (2.1) over disorder $\mathbf{E}_{n}$, the searched Bose unconditional canonical occupancy probability clearly is

$$
(2.3) \mathbb{P}\left(K_{n, k}(m)=k_{m} ; m=1, . ., n\right)=\mathbb{E P}_{\mathbf{E}_{n}}\left(K_{n, k}(m)=k_{m} ; m=1, . ., n\right) .
$$

As a symmetric function of the $k_{m}$, this annealed distribution is exchangeable. In particular, $\mathbb{E}\left(K_{n, k}(m)\right)=k / n, m=1, . ., n$. Further, for instance, with $k_{1} \in\{0, . ., k\}$ and $\mathbf{E}_{n \backslash 1}:=\left(E_{2}, . ., E_{n}\right)$

$$
\begin{aligned}
\mathbb{P}_{\mathbf{E}_{n}}\left(K_{n, k}(1)=k_{1}\right) & =\frac{e^{-\beta E_{1} k_{1}}}{Z_{k, \mathbf{E}_{n}}(\beta)} \sum_{k_{2}+. .+k_{n}=k-k_{1}} \prod_{m=2}^{n} e^{-\beta E_{m} k_{m}}, \\
& =\frac{e^{-\beta E_{1} k_{1}} Z_{k-k_{1}, \mathbf{E}_{n \backslash 1}}(\beta)}{Z_{k, \mathbf{E}_{n}}(\beta)}
\end{aligned}
$$

and

$$
\mathbb{P}\left(K_{n, k}(1)=k_{1}\right)=\mathbb{E}\left[\frac{e^{-\beta E_{1} k_{1}} Z_{k-k_{1}, \mathbf{E}_{n \backslash 1}}(\beta)}{Z_{k, \mathbf{E}_{n}}(\beta)}\right]
$$

is the one-dimensional marginal of $K_{n, k}(m)=k_{m} ; m=1, . ., n$. Except for very particular cases as in the example which follows, a closed-form expression of Eq. (2.3) seems out of reach.

Example: As a limiting example of Bose distribution, when $\beta \downarrow 0$, the joint law of $K_{n, k}(m) ; m=1, . ., n$ tends to the uniform Bose-Einstein distribution (see Feller [10], and Holst, [11])

$$
\mathbb{P}\left(K_{n, k}(m)=k_{m} ; m=1, . ., n\right)=\frac{1}{\left(\begin{array}{c}
n+k-1 \\
k
\end{array}\right)}
$$

and the 1-dimensional distribution at high temperature reads

$$
\mathbb{P}\left(K_{n, k}(1)=k_{1}\right)=\frac{\left(\begin{array}{c}
n+k-k_{1}-2 \\
k-k_{1}
\end{array}\right)}{\left(\begin{array}{c}
n+k-1 \\
k
\end{array}\right)}, k_{1}=0, . ., k
$$


Before investigating some additional statistical properties of the Bose distribution displayed in Eq. (2.3), let us first stress immediate consequences of the model.

- Energy of a single particle: Assume first that $k=1$. Then the sample is made of a single particle. From Bose law, this particle will launch on energy level number $m$ with conditional probability $e^{-\beta E_{m}} / Z_{\mathbf{E}_{n}}(\beta)$ where $Z_{\mathbf{E}_{n}}(\beta):=Z_{1, \mathbf{E}_{n}}(\beta)=\sum_{1}^{n} e^{-\beta E_{m}}$. The corresponding energy attached to this single particle, say $\mathcal{E}_{n}$, has conditional law given by

$$
\mathbb{P}_{\mathbf{E}_{n}}\left(\mathcal{E}_{n}=E_{m}\right)=e^{-\beta E_{m}} / Z_{\mathbf{E}_{n}}(\beta)=: V_{m, n}(\beta), m=1, . ., n .
$$

The random probabilities $V_{m, n}(\beta)$ satisfy $\sum_{1}^{n} V_{m, n}(\beta) \stackrel{\text { a.s. }}{=} 1$ and so constitute a random partition of unity. Let now $\mathbb{E}_{\mathbf{E}_{n}}\left(e^{-\lambda \mathcal{E}_{n}}\right)$ be the conditional LaplaceStieltjes transform $(\mathrm{LST})$ of $\mathcal{E}_{n}$. Then $\mathbb{E}_{\mathbf{E}_{n}}\left(e^{-\lambda \mathcal{E}_{n}}\right)=Z_{\mathbf{E}_{n}}(\lambda+\beta) / Z_{\mathbf{E}_{n}}(\beta)$. Averaging over $\mathbf{E}_{n}$

$$
\mathbb{E}\left(e^{-\lambda \mathcal{E}_{n}}\right):=\mathbb{E} \mathbb{E}_{\mathbf{E}_{n}}\left(e^{-\lambda \mathcal{E}_{n}}\right)=\mathbb{E}\left[Z_{\mathbf{E}_{n}}(\lambda+\beta) / Z_{\mathbf{E}_{n}}(\beta)\right]
$$

characterizes the distribution of the single particle energy. In particular,

$$
\mathbb{E}\left(\mathcal{E}_{n}\right)=\partial_{\beta} \mathbb{E}\left[-\log Z_{\mathbf{E}_{n}}(\beta)\right] .
$$

In this energy-biased picking procedure $\mathbf{E}_{n} \rightarrow \mathcal{E}_{n}$, states with low energy levels are clearly favored. One therefore expects $\mathcal{E}_{n}$ to be stochastically smaller than the typical energy level $E_{n}$ from the iid sequence $\mathbf{E}_{n}$.

To study the Sherrington-Kirkpatrick model for spin glasses, similar questions were raised for different purposes by Derrida in the early eighties, under the (ruled out here) hypothesis that energies were normally distributed (see [5]$[7])$.

- Total energy: Let now $\mathcal{H}_{n, k}:=\sum_{m=1}^{n} E_{m} K_{n, k}(m)$ stand for total energy of the $k$ particles system where occupancies law is as in Eq. (2.1); its LST is clearly given by

$$
\mathbb{E}\left[e^{-\lambda \mathcal{H}_{n, k}}\right]=\mathbb{E}\left[\frac{Z_{k, \mathbf{E}_{n}}(\lambda+\beta)}{Z_{k, \mathbf{E}_{n}}(\beta)}\right], \lambda \geq 0 .
$$

Let us now show that, when the number of energy levels is fixed, the proportions of particles tend to concentrate on the ground state as the number of particles increases.

Proposition 1 For each $m \in\{1, . ., n\}$, let $\mathbf{E}_{n \backslash m}:=\left\{E_{1}, . . E_{m-1}, E_{m+1}, . . E_{n}\right\}$. With $n$ fixed, as the number of particles grows, conditionally given $\mathbf{E}_{n}$, we have

$$
\left(\frac{K_{n, k}(m)}{k} ; m=1, . ., n\right) \underset{k \uparrow \infty}{\longrightarrow}\left(V_{m, n}:=\mathbf{I}_{\left\{E_{m}<\mathbf{E}_{n \backslash m}\right\}} ; m=1, . ., n\right)
$$


in distribution.

Proof: Let us first consider the ordered version $\mathbf{E}_{(n)}$ of the energy sequence $\mathbf{E}_{n}$, namely: $\mathbf{E}_{(n)}:=\left(E_{(1), n}, . ., E_{(n), n}\right)$ with $E_{(1), n}<. .<E_{(n), n}$. Let also $\widetilde{E}_{(m), n}:=E_{(m), n}-E_{(1), n}>0, m=1, . ., n$. Developing the product partition function

$$
\prod_{m=1}^{n}\left(1-z e^{-\beta E_{m}}\right)^{-1}=\prod_{m=1}^{n}\left(1-z e^{-\beta E_{(m), n}}\right)^{-1}
$$

appearing in Eq. (2.2) into a sum of rational fractions, extracting its $z^{k}$-coefficient and isolating the ground-state term, we get

$$
\begin{gathered}
Z_{k, \mathbf{E}_{n}}(\beta)=Z_{k, \mathbf{E}_{(n)}}(\beta)= \\
e^{-\beta E_{(1), n} k}\left(\prod_{m \neq 1} \frac{1}{1-e^{-\beta \widetilde{E}_{(m), n}}}+\sum_{m=2}^{n} e^{-\beta \widetilde{E}_{(m), n} k} \prod_{l \neq m} \frac{1}{1-e^{-\beta\left(E_{(l), n}-E_{(m), n}\right)}}\right) .
\end{gathered}
$$

Inserting this expression into Eq. (2.1), with $K_{(n), k}(m) ; m=2, . ., n$ the occupancies of energy levels $E_{(2), n}, . ., E_{(n), n}$ and $k_{m} \in \mathbb{N}_{0} ; m=2, . ., n$, such that $\sum_{m=2}^{n} k_{m} \leq k$, we get

$$
\begin{gathered}
\mathbb{P}_{\mathbf{E}_{(n)}}\left(K_{(n), k}(m)=k_{m} ; m=2, . ., n\right)= \\
(1-\varepsilon(k)) \prod_{m=2}^{n}\left\{e^{-\beta \widetilde{E}_{(m), n} k_{m}}\left(1-e^{-\beta \widetilde{E}_{(m), n}}\right)\right\}
\end{gathered}
$$

where $\varepsilon(k)=e^{-\beta \widetilde{E}_{(2), n} k} \prod_{l \neq 1}\left(1-e^{-\beta \widetilde{E}_{(l), n}}\right) / \prod_{l \neq 2}\left(1-e^{-\beta\left(E_{(l), n}-E_{(2), n}\right)}\right)$ is a negative corrective term that goes to 0 exponentially fast with $k$ becoming large. Suppose $k_{m}=\left\lfloor k s_{m}\right\rfloor$ for some fixed $s_{m} \in(0,1] ; m=2, . ., n$. In this case, the probability displayed in Eq. (2.5) goes to 0 when $k$ goes to $\infty$ : in other words, the probabilities of $K_{(n), k}(m) / k ; m=2, . ., n$ all concentrate on 0 and therefore all the probability mass goes to ground state $(m=1)$. This is statement in Eq. (2.4) where $\mathbf{I}_{\{A\}}$ stands for the set indicator of the event $A$. Note that $\left(V_{m, n}:=\mathbf{I}_{\left\{E_{m}<\mathbf{E}_{n \backslash m}\right\}} ; m=1, . ., n\right)$ is an exchangeable partition of unity. In particular, for each $m, \mathbb{E}\left(V_{m, n}\right)=1 / n$ and $\sigma^{2}\left(V_{m, n}\right)=(1-1 / n) / n$.

- Some remarks on Maxwell-Boltzmann version of the occupancy problem: This limiting behavior of occupancies is in sharp contrast with the one arising in a Maxwell-Boltzmann version of the randomized occupancy problem. Let us briefly address this point.

With $\sum_{1}^{n} V_{m, n}(\beta) \stackrel{a . s .}{=} 1$, the random numbers $V_{m, n}(\beta)=\frac{e^{-\beta E_{m}}}{\sum_{1}^{n} e^{-\beta E_{m}}}$ have been shown to be the conditional Bose probabilities that a single particle hits state $m$ with energy $E_{m}$. The partitioning system $\mathbf{V}_{n}(\beta):=\left(V_{1, n}(\beta), . ., V_{n, n}(\beta)\right)$ 
constitute an exchangeable random partition of unity (in particular, for each $m$, $\left.\mathbb{E}\left(V_{m, n}(\beta)\right)=1 / n\right)$. The random quantities $\mathbf{V}_{n}(\beta)$ are essential in the derivation of the Maxwell-Boltzmann sampling distribution version of the randomized occupancy problem. It proceeds as follows: let $\left(U_{1}, . ., U_{k}\right)$ be $k$ iid uniform throws on $[0,1]$ partitioned by $\mathbf{V}_{n}(\beta)$. Let $\left(B_{n, k}(1), . ., B_{n, k}(n)\right)$ be an integralvalued random vector which counts the number of visits to the different energy levels in a $k$-sample in the following sense: if $M_{l}$ is the random state label which the $l$-th trial hits, then $B_{n, k}(m):=\sum_{l=1}^{k} \mathbf{I}_{\left\{M_{l}=m\right\}}, m=1, . ., n$. Clearly $\mathbb{P}_{\mathbf{V}_{n}(\beta)}\left(M_{l}=m\right)=V_{m, n}(\beta)$ and, given $\mathbf{V}_{n}(\beta)$, state $m$ is chosen proportionally to its "size" $V_{m, n}(\beta)$.

With $\left(b_{1}, . ., b_{n}\right) \in \mathbb{N}_{0}^{n}$, satisfying $\sum_{m=1}^{n} b_{m}=k,\left(B_{n, k}(m)=b_{m} ; m=1, . ., n\right)$ now follows the conditional multinomial distribution:

$$
\mathbb{P}_{\mathbf{V}_{n}(\beta)}\left(B_{n, k}(m)=b_{m} ; m=1, . ., n\right)=\frac{k !}{\prod_{m=1}^{n} b_{m} !} \prod_{m=1}^{n} V_{m, n}^{b_{m}}(\beta) .
$$

Averaging over $\mathbf{V}_{n}(\beta)$ gives the Maxwell-Boltzmann distribution

$$
\mathbb{P}\left(B_{n, k}(m)=b_{m} ; m=1, . ., n\right)=\mathbb{E} \mathbb{P}_{\mathbf{V}_{n}(\beta)}\left(B_{n, k}(m)=b_{m} ; m=1, . ., n\right) .
$$

Clearly, proceeding in this way to fill up sequentially the energy levels, particles are assumed distinguishable and, by strong law of large numbers, conditionally given $\mathbf{E}_{n}$

$$
\left(\frac{B_{n, k}(m)}{k} ; m=1, . ., n\right) \underset{k \uparrow \infty}{\longrightarrow}\left(V_{m, n}(\beta) ; m=1, . ., n\right)
$$

almost surely and in distribution (compare with Eq. (2.4)).

Note that computational problems also arise in the canonical ensemble, since, averaging over $\mathbf{E}_{n}$, one needs to evaluate the awkward quantities

$$
\mathbb{E}\left[\prod_{m=1}^{n} V_{m, n}^{b_{m}}(\beta)\right]=\mathbb{E}\left[\frac{e^{-\beta \sum_{m=1}^{n} E_{m} b_{m}}}{\left(\sum_{m=1}^{n} e^{-\beta E_{m}}\right)^{k}}\right] .
$$

Due to these computational difficulties, canonical randomized Maxwell Boltzmann statistics turns out to be as intricate as Bose one. We shall not enter into more details on this question and rather focus on the Bose occupancy problem as defined by Eqs. (2.1-2.2).

- Random allocation scheme representation of Bose distribution: From Eqs. (2.1-2.2), we observe immediately the distributional identity:

$$
\left(K_{n, k}(m)=k_{m} ; m=1, . ., n\right) \stackrel{d}{=}\left(\xi_{1}, . ., \xi_{n} \mid \zeta_{n}=k\right)
$$

where $\left(\xi_{1}, . ., \xi_{n}\right)$ is iid on $\mathbb{N}_{0}^{n}$ conditioned by its $\operatorname{sum} \zeta_{n}:=\sum_{1}^{n} \xi_{m}=k$ and (2.7) $\mathbb{P}\left(\xi_{1}=k_{1}\right)=\mathbb{E}\left[\mathbb{P}_{\mathbf{E}_{n}}\left(\xi_{1}=k_{1}\right)\right]=\mathbb{E}\left[e^{-\beta E_{1} k_{1}}\left(1-e^{-\beta E_{1}}\right)\right], k_{1} \in \mathbb{N}_{0}$, 
a mixture of geometric distribution with success probability $e^{-\beta E_{1}}$. Such a conditional representation of the occupancies is called a random allocation scheme property in Kolchin, [13].

Let $\rho>0$ be the density of particles per state and let $\kappa_{n}:=\lfloor n \rho\rfloor$.

Proposition 2 Assume $\mathbb{E}\left[\left(e^{\alpha+\beta E_{1}}-1\right)^{-1}\right]=\rho$ admits a solution for $\alpha:=$ $\alpha(\beta, \rho)>0$. Then

$$
K_{n, \kappa_{n}}(1) \stackrel{d}{\rightarrow}_{n \uparrow \infty} \mathcal{K}_{\rho},
$$

where, with $\alpha:=\alpha(\beta, \rho), \mathcal{K}_{\rho}$ is a mean- $\rho$ mixture of geometric distribution with probability generating function

$$
\mathbb{E}\left[u^{\mathcal{K}_{\rho}}\right]=\mathbb{E}\left[\frac{1-e^{-\left(\alpha+\beta E_{1}\right)}}{1-u e^{-\left(\alpha+\beta E_{1}\right)}}\right], u \in[0,1] .
$$

Proof: This follows from the above allocation representation property and the Gibbs conditioning principle. The random variable $\mathcal{K}_{\rho}$ is the asymptotic typical cell occupation at density $\rho$ of particles per state. Its law can be obtained by exponential-tilting the ones of $\xi_{m}, m=1, . ., n$ namely, with $\mathbb{P}_{\mathbf{E}_{n}}\left(\xi_{m}=k_{m}\right)=$ $e^{-\beta E_{m} k_{m}}\left(1-e^{-\beta E_{m}}\right)$, by considering :

$$
\mathbb{P}\left(\widetilde{\xi}_{m, \alpha}=k_{1}\right):=\mathbb{E}\left(\mathbb{P}_{\mathbf{E}_{n}}\left(\widetilde{\xi}_{m, \alpha}=k_{m}\right)\right)=\mathbb{E}\left[\frac{e^{-\alpha k_{m}} \mathbb{P}_{\mathbf{E}_{n}}\left(\xi_{m}=k_{m}\right)}{\sum_{k_{m} \geq 0} e^{-\alpha k_{m}} \mathbb{P}_{\mathbf{E}_{n}}\left(\xi_{m}=k_{m}\right)}\right]
$$

Given $\mathbf{E}_{n}, \widetilde{\xi}_{m, \alpha}, m=1, . ., n$ are therefore geometrically distributed with iid success random probabilities $e^{-\left(\alpha+\beta E_{m}\right)}$ and expected values $\left(e^{\alpha+\beta E_{m}}-1\right)^{-1}$. Applying Gibbs conditioning principle to obtain Eq. (2.8), one needs to adjust $\alpha$ so as to meet the conditioning requirement $\widetilde{\zeta}_{n, \alpha}:=\sum_{m=1}^{n} \widetilde{\xi}_{m, \alpha}=\lfloor n \rho\rfloor$. This will be possible at the only condition that there exists an $\alpha>0$ such that

$$
\mathbb{E}\left[\mathcal{K}_{\rho}\right]=\mathbb{E}\left[\left(e^{\alpha+\beta E_{1}}-1\right)^{-1}\right]=\rho .
$$

This condition will appear more clearly later to separate, in some cases, between a fluid and a condensed phase. In case there is no $\alpha$ for a given $\rho$ (see below for examples in the condensed phase), there is no typical limiting cell occupation at density $\rho$.

- Gibbs randomization of sample size (variable particle number): As it was stressed above, the canonical conditional distributions given sample size is $k$ are extremely difficult to evaluate in general (even when $k=1$ ). To circumvent this drawback, we shall now assume the number of particles is variable and so randomize sample size. Let $\alpha>0$ ( $\alpha=\beta \mu$ where $\mu>0$ is fugacity). Assume the 
number of particles $K_{n, \alpha}$ is now random with law given by the Gibbs probability measure

$$
\mathbb{P}_{\mathbf{E}_{n}}\left(K_{n, \alpha}=k\right)=\frac{e^{-\alpha k} Z_{k, \mathbf{E}_{n}}(\beta)}{Z_{\mathbf{E}_{n}}(\alpha, \beta)}
$$

where the grand canonical partition now reads

$$
Z_{\mathbf{E}_{n}}(\alpha, \beta)=\sum_{k \geq 0} e^{-\alpha k} Z_{k, \mathbf{E}_{n}}(\beta)=\prod_{m=1}^{n} \frac{1}{1-e^{-\left(\alpha+\beta E_{m}\right)}} .
$$

Alternatively, with $\lambda \geq 0$, we have

$$
\begin{aligned}
\mathbb{E}_{\mathbf{E}_{n}}\left(e^{-\lambda K_{n, \alpha}}\right) & =\frac{Z_{\mathbf{E}_{n}}(\alpha+\lambda, \beta)}{Z_{\mathbf{E}_{n}}(\alpha, \beta)}=\prod_{m=1}^{n} \frac{1-e^{-\left(\alpha+\beta E_{m}\right)}}{1-e^{-\left(\lambda+\alpha+\beta E_{m}\right)}} \\
\mathbb{E}\left(e^{-\lambda K_{n, \alpha}}\right) & =\left[\mathbb{E}\left(\frac{1-e^{-\left(\alpha+\beta E_{1}\right)}}{1-e^{-\left(\lambda+\alpha+\beta E_{1}\right)}}\right)\right]^{n} .
\end{aligned}
$$

Given $\mathbf{E}_{n}$, the expected number of particles can be obtained through:

$$
\mathbb{E}_{\mathbf{E}_{n}}\left(K_{n, \alpha}\right)=-\partial_{\alpha} \log Z_{\mathbf{E}_{n}}(\alpha, \beta) .
$$

Indexing now cell occupancies by $\alpha$ rather than $k$, with $k_{m} \in \mathbb{N}_{0} ; m=1, . ., n$, the joint occupancies probability reads

$$
\mathbb{P}_{\mathbf{E}_{n}}\left(K_{n, \alpha}(m)=k_{m} ; m=1, . ., n\right)=\frac{1}{Z_{\mathbf{E}_{n}}(\alpha, \beta)} \prod_{m=1}^{n} e^{-\left(\alpha+\beta E_{m}\right) k_{m}},
$$

and $K_{n, \alpha}:=\sum_{m=1}^{n} K_{n, \alpha}(m)$.

Let $\phi_{1}(\beta):=\mathbb{E} e^{-\beta E_{1}}$ be the Laplace-Stieltjes transform of $E_{1}$. Averaging over $\mathbf{E}_{n}$, the unconditional occupancy probability is

$$
\begin{gathered}
\mathbb{P}\left(K_{n, \alpha}(m)=k_{m} ; m=1, . ., n\right):=\mathbb{E P}_{\mathbf{E}_{n}}\left(K_{n, \alpha}(m)=k_{m} ; m=1, . ., n\right)= \\
\mathbb{E}\left[\prod_{m=1}^{n} e^{-\left(\alpha+\beta E_{m}\right) k_{m}}\left(1-e^{-\left(\alpha+\beta E_{m}\right)}\right)\right]=\prod_{m=1}^{n} \mathbb{E}\left[e^{-\left(\alpha+\beta E_{1}\right) k_{m}}\left(1-e^{-\left(\alpha+\beta E_{1}\right)}\right)\right] \\
(2.13) \quad=\prod_{m=1}^{n}\left(e^{-\alpha k_{m}}\left[\phi_{1}\left(\beta k_{m}\right)-e^{-\alpha} \phi_{1}\left(\beta\left(k_{m}+1\right)\right)\right]\right) .
\end{gathered}
$$

It is exchangeable and under the form of a mere product measure. In particular, with $k_{1} \in \mathbb{N}_{0}$

$$
\mathbb{P}\left(K_{n, \alpha}(1)=k_{1}\right)=e^{-\alpha k_{1}}\left[\phi_{1}\left(\beta k_{1}\right)-e^{-\alpha} \phi_{1}\left(\beta\left(k_{1}+1\right)\right)\right] .
$$


Stated differently, with $\xi_{1, \alpha}$ the $\mathbb{N}_{0}$-valued random variable with distribution $\mathbb{P}\left(\xi_{1, \alpha} \geq k_{1}\right)=e^{-\alpha k_{1}} \phi_{1}\left(\beta k_{1}\right)$, we have

$$
\mathbb{P}\left(K_{n, \alpha}(m)=k_{m} ; m=1, . ., n\right)=\prod_{m=1}^{n} \mathbb{P}\left(\xi_{m, \alpha}=k_{m}\right)
$$

where $\left(\xi_{1, \alpha}, . ., \xi_{n, \alpha}\right)$ is an iid sequence. This factorized representation turns out to be useful.

1/ For example, let $K_{n, \alpha}^{+}$be the grand-canonical occupancy of state with the largest amount of particles. Then

$$
\mathbb{P}\left(K_{n, \alpha}^{+} \leq k\right)=\left(1-e^{-\alpha k} \phi_{1}(\beta k)\right)^{n}
$$

and one expects some Poisson approximation. However, following Embrechts et al [8], Theorem 3.1 .3 , p. 117, observing that

$$
\frac{\mathbb{P}\left(\xi_{1, \alpha} \geq k_{1}\right)}{\mathbb{P}\left(\xi_{1, \alpha} \geq k_{1}-1\right)} \leq e^{-\alpha} \in(0,1) \text { and } \lim _{k_{1} \uparrow \infty} \frac{\mathbb{P}\left(\xi_{1, \alpha} \geq k_{1}\right)}{\mathbb{P}\left(\xi_{1, \alpha} \geq k_{1}-1\right)}<1,
$$

there is no sequence $k_{n}$ such that $n e^{-\alpha k_{n}} \phi_{1}\left(\beta k_{n}\right) \rightarrow \gamma \in(0, \infty)$. Therefore, whatever the sequence $k_{n}$ is, there is no non degenerate limit of the form $\mathbb{P}\left(K_{n, \alpha}^{+} \leq k_{n}\right) \rightarrow x$ where $x \in[0,1] \backslash\{0,1\}$; in other words, for every sequence $k_{n}$ such that $\mathbb{P}\left(K_{n, \alpha}^{+} \leq k_{n}\right) \rightarrow x$, either $x=0$ or 1 and there is no way to normalize $K_{n, \alpha}^{+}$so as to obtain a non-degenerate limit distribution. The discreteness of the distributions involved prevents the maximum from converging properly to some extreme value distribution and instead forces this oscillatory behavior.

2/ The grand canonical distribution of total energy: Let us consider the LST of the random variable $\mathcal{H}_{n, \alpha}$. With $\lambda \geq 0$ and $k_{m} \in \mathbb{N}_{0}$, it is

$$
\begin{gathered}
\mathbb{E}\left(e^{-\lambda \mathcal{H}_{n, \alpha}}\right)=\mathbb{E}\left(\sum_{k_{m} ; m=1, . ., n} e^{-\lambda \sum_{m=1}^{n} k_{m} E_{m}} \mathbb{P}_{\mathbf{E}_{n}}\left(K_{n, \alpha}(m)=k_{m} ; m=1, . ., n\right)\right) \\
=\mathbb{E}\left(\prod_{m=1}^{n} \sum_{k_{m}} e^{-\left(\alpha+(\lambda+\beta) E_{m}\right) k_{m}}\left(1-e^{-\left(\alpha+\beta E_{m}\right)}\right)\right) \\
=\mathbb{E}\left(\prod_{m=1}^{n} \frac{1-e^{-\left(\alpha+\beta E_{m}\right)}}{1-e^{-\left(\alpha+(\lambda+\beta) E_{m}\right)}}\right)=\left\{\mathbb{E}\left(\frac{1-e^{-\left(\alpha+\beta E_{1}\right)}}{1-e^{-\left(\alpha+(\lambda+\beta) E_{1}\right)}}\right)\right\}^{n} .
\end{gathered}
$$

This shows that $\mathcal{H}_{n, \alpha}$ is the sum of $n$ iid random variables whose increment has LST given by $\mathbb{E}\left(\frac{1-e^{-\left(\alpha+\beta E_{1}\right)}}{1-e^{-\left(\alpha+(\lambda+\beta) E_{1}\right)}}\right)$. In particular, by strong law of large 
numbers, $\mathcal{H}_{n, \alpha} / n$ converges a.s. to the mean energy per state, namely

$$
\frac{\mathcal{H}_{n, \alpha}}{n} \rightarrow_{n \uparrow \infty} \mathbb{E}\left(\frac{E_{1}}{e^{\alpha+\beta E_{1}}-1}\right) .
$$

\subsection{A condensation phenomenon}

The origin of the condensation phenomenon to be described now results from the conjunction of two main effects of the model under study: the randomness of the energy levels (the system is disordered) and the indistinguishability of particles to be allocated. Phase transitions induced by disorder is well-known in related contexts (see [14]).

- A Bose-Einstein like condensation phenomenon: It remains to interpret $\alpha$ in more details. Observing that

$$
\mathbb{E}_{\mathbf{E}_{n}}\left(K_{n, \alpha}(m)\right)=-\frac{1}{\beta} \partial_{E_{m}} \log Z_{\mathbf{E}_{n}}(\alpha, \beta)=\frac{1}{e^{\alpha+\beta E_{m}}-1}
$$

we have

$$
\begin{aligned}
\mathbb{E}_{\mathbf{E}_{n}}\left(K_{n, \alpha}\right) & =\sum_{m=1}^{n} \frac{1}{e^{\alpha+\beta E_{m}}-1} \\
\nu & : \quad=\mathbb{E} \mathbb{E}_{\mathbf{E}_{n}}\left(K_{n, \alpha}\right)=n \mathbb{E}\left(\left(e^{\alpha+\beta E_{1}}-1\right)^{-1}\right) .
\end{aligned}
$$

Assuming the number of levels $n$ to be the extensive variable of such a mean-field model, we thus get the particle density per state

$$
\rho:=\frac{\nu}{n}=\mathbb{E}\left(\left(e^{\alpha+\beta E_{1}}-1\right)^{-1}\right) .
$$

Usually, it is therefore of interest to try to deduce $\alpha$ from $(\rho, \beta)$ which are the physical quantities which are known in practice. Letting $z:=e^{-\alpha}$ denote fugacity, developing the argument of the expectation, this is also the implicit state equation

$$
\rho=z h_{\beta}(z)
$$

where $h_{\beta}(z)$ has the power series representation

$$
h_{\beta}(z)=\sum_{i \geq 0} z^{i} h_{i}(\beta) \text { with } h_{i}(\beta)=\phi_{1}(\beta(i+1)) .
$$

Let $z_{c}>0$ be the convergence radius of the series

$$
H_{\beta}(z):=z h_{\beta}(z)=\sum_{i \geq 1} z^{i} \phi_{1}(\beta i) .
$$


Two cases arise: $z_{c}=1$ or $z_{c}>1$. If $z_{c}>1, h_{\beta}(1)$ exists, is monotone decreasing and maps $\beta \in(0, \infty)$ onto $(\infty, 0)$; let therefore $\beta_{c}$ be defined by $\rho=h_{\beta_{c}}(1)$. If $z_{c}=1$, let $\beta_{c}$ be defined as before if $H_{\beta}\left(z_{c}\right)<\infty$ and $\beta_{c}:=\infty$ if $H_{\beta}\left(z_{c}\right)=\infty$. In any case, for $\beta<\beta_{c}$, by Bürmann-Lagrange inversion formula, $\alpha=-\log z$ where $z \in\left(0,1 \wedge z_{c}\right)$ and

$$
z=1+\sum_{l \geq 1} \frac{\rho^{l}}{l} h_{l}(\beta) \text { with } h_{l}(\beta):=\left[z^{l-1}\right] h_{\beta}(z)^{-l}
$$

A Bose-Einstein condensation-like phenomenon therefore pops in when the probability that energies take values close to 0 are small enough. We shall now supply three examples.

- Examples: In example $(i)$, the probability mass in 0 of energy is chosen log-algebraically large. In the subsequent example $(i i)$, the probability mass of energy in 0 is exponentially small. These two examples show that a condensation like phenomenon occurs if the probability distribution of energy in a neighborhood of 0 is small enough (with growth at least linear). In a last extreme example (iii), the support of the law of $E_{1}$ is $\left(\epsilon_{0}, \infty\right), \epsilon_{0}>0$; ground state $\epsilon_{0}$ is away from 0 with probability 1 .

(i) Assume the distribution of energy $E_{1}$, say $F_{1}(\epsilon):=\int^{\epsilon} f_{1}\left(\epsilon^{\prime}\right) d \epsilon^{\prime}$, satisfies:

$$
F_{1}(\epsilon) \sim_{\epsilon \downarrow 0} C \cdot \epsilon^{\theta} / L(1 / \epsilon)
$$

where $C>0, \theta \geq 0$ and $L(x)$ a slowly varying at $\infty$ function ( $\operatorname{such}$ as $\log x$ ). The smaller $\theta$ is, the more energy is concentrated at 0 . Then by KaramataTauberian Theorem (see Bingham et al, [2])

$$
\phi_{1}(\beta) \sim_{\beta \uparrow \infty} C \cdot \Gamma(1+\theta) \cdot \beta^{-\theta} / L(\beta)
$$

and $\phi_{1}(\beta)$ is regularly varying at $\infty$. For all $\theta \geq 0, \beta>0$, the convergence radius of $H_{\beta}(z)$ is $z_{c}=1$. If $\theta \leq 1, H_{\beta}\left(z_{c}\right)=\infty$ whereas for $\theta>1, H_{\beta}\left(z_{c}\right)<\infty$. If $\theta \leq 1$, there is no condensation whereas when $\theta>1$, a critical value $\beta_{c}$ of $\beta$ can be defined. When $\theta>1$, the order of differentiability of $z \rightarrow H_{\beta}(z)$ at $z_{c}=1$ is $\lfloor\theta\rfloor-1$.

In all such cases, $\phi_{1}(2 \beta) / \phi_{1}(\beta) \sim_{\beta \uparrow \infty} 2^{-\theta}$ does not tend to 0 when $\beta \uparrow \infty$.

(ii) Assume density of $E_{1}$ satisfies: $f_{1}(\epsilon) \sim_{\epsilon \downarrow 0} C \cdot \epsilon^{-3 / 2} e^{-1 / \epsilon}$ : a small value of $E_{1}$ is rather unlikely (exponentially small). Then, $\phi_{1}(\beta) \sim_{\beta \uparrow \infty} C \cdot e^{-\sqrt{\beta}}$ so that $z_{c}=1$ with $H_{\beta}\left(z_{c}\right)<\infty$. In this case, $\phi_{1}(2 \beta) / \phi_{1}(\beta)$ does tend to 0 when $\beta \uparrow \infty$.

This example is a particular case of the related one for which: $f_{1}(\epsilon) \sim_{\epsilon \downarrow 0} C$. $e^{-1 / \epsilon^{c}}$ for some $c>0$. In this case, a small value of $E_{1}$ is exp-algebraically small 
and, by applying the saddle point method: $\phi_{1}(\beta) \sim_{\beta \uparrow \infty} C \cdot \beta^{-\frac{c+2}{2(c+1)}} e^{-B \cdot \beta^{c /(c+1)}}$ for some constant $B>0$. Here again, $\phi_{1}(2 \beta) / \phi_{1}(\beta) \rightarrow 0$ when $\beta \uparrow \infty$.

(iii) Let $\epsilon_{0}>0$ denote spectral gap and $a>0$. Assume $f_{1}(\epsilon)=a e^{-a\left(\epsilon-\epsilon_{0}\right)}$, $\epsilon>\epsilon_{0}$ (a truncated exponential model). Then $\phi_{1}(\beta)=\frac{e^{-\beta \epsilon_{0}}}{1+\beta / a}$. Here $z_{c}=e^{\beta \epsilon_{0}}>$ 1 and $H_{\beta}\left(z_{c}\right)=\infty$. Note that $H_{\beta}(z)$ is infinitely differentiable (smooth) at $z=1<z_{c}$. In this case, $\beta_{c}$ is uniquely determined by $\sum_{i \geq 1} \frac{e^{-\beta_{c} \epsilon_{0} i}}{1+\left(\beta_{c} i\right) / a}=\rho$.

Remark: In this approach, the free parameter is density $\rho$ and the Lagrange inversion formula only holds for $\beta<\beta_{c}$ (temperature is high enough). If $\beta$ is the free parameter, Lagrange inversion formula only is valid for $\rho<\rho_{c}:=h_{\beta}$ (1) (the free Bose gas has small enough density). In the $(\beta, \rho)$ plane, the critical line $\rho_{c}=: \rho_{c}(\beta)$ separates a diluted fluid phase $\rho<\rho_{c}$ from a condensed phase $\rho>\rho_{c}$. Note that $\rho_{c}(\beta)=\mathbb{E}\left(\left(e^{\beta E_{1}}-1\right)^{-1}\right) \cdot \diamond$

One can summarize shortly the results as follows:

Proposition 3 We have:

(i) If $\mathbb{E}\left(1 / E_{1}\right)=\infty$, there is no condensation and the only available phase is fluid.

(ii) If $\mathbb{E}\left(1 / E_{1}\right)<\infty$, there is condensation: the critical line separating the condensed $\left(\rho>\rho_{c}\right)$ from fluid $\left(\rho<\rho_{c}\right)$ phases has equation $\rho=\rho_{c}(\beta)=$ $\mathbb{E}\left(\left(e^{\beta E_{1}}-1\right)^{-1}\right)$ which is also the convergent series

$$
\rho=\rho_{c}(\beta)=\sum_{i \geq 1} \phi_{1}(\beta i)
$$

involving the LST of energies.

- Thermodynamics of the diluted fluid phase: Internal energy reads $U_{n}(\alpha, \beta)=$ $\mathbb{E}\left(U_{\mathbf{E}_{n}}(\alpha, \beta)\right)$ where

$$
U_{\mathbf{E}_{n}}(\alpha, \beta)=-\partial_{\beta} \log Z_{\mathbf{E}_{n}}(\alpha, \beta)=\sum_{m=1}^{n} \frac{E_{m}}{e^{\alpha+\beta E_{m}}-1} .
$$

Observing that $\mathbb{E}\left(\frac{E_{1}}{e^{\alpha+\beta E_{1}}-1}\right)<\infty$ we get

$$
\frac{U_{\mathbf{E}_{n}}(\alpha, \beta)}{n} \underset{n \uparrow \infty}{\underset{a . s .}{\longrightarrow}} \mathbb{E}\left(\frac{E_{1}}{e^{\alpha+\beta E_{1}}-1}\right)=: u(\alpha, \beta) .
$$

Further, $U_{n}(\alpha, \beta)=n \cdot u(\alpha, \beta)$. The quantity $u(\alpha, \beta)$ is internal energy per state. 
Assume $\mathbb{E} \log \left(1-e^{-\left(\alpha+\beta E_{1}\right)}\right)<\infty$ for each $(\alpha, \beta) \in \mathbb{R}_{+}^{2}$. With $N_{n}(\epsilon):=$ $\sum_{m=1}^{n} \mathbf{I}_{\left\{E_{m} \leq \epsilon\right\}}$, let the empirical distribution function (or density of states) of $\mathbf{E}_{n}$ be $D_{n}(\epsilon):=n^{-1} N_{n}(\epsilon)$. Let $F_{\mathbf{E}_{n}}(\alpha, \beta):=-\frac{1}{\beta} \log Z_{\mathbf{E}_{n}}(\alpha, \beta)$ be the conditional free energy. Then, by the strong law of large numbers

$$
\begin{gathered}
-\frac{1}{n} F_{\mathbf{E}_{n}}(\alpha, \beta)=\frac{1}{n \beta} \sum_{m=1}^{n} \log \left(1-e^{-\left(\alpha+\beta E_{m}\right)}\right)= \\
\frac{1}{\beta} \int_{\mathbb{R}_{+}} \log \left(1-e^{-(\alpha+\beta \epsilon)}\right) D_{n}(d \epsilon) \underset{n \uparrow \infty}{\stackrel{a . s .}{\longrightarrow}} \frac{1}{\beta} \mathbb{E} \log \left(1-e^{-\left(\alpha+\beta E_{1}\right)}\right)=: f(\alpha, \beta)
\end{gathered}
$$

and

$$
F_{n}(\alpha, \beta):=\mathbb{E}\left(F_{\mathbf{E}_{n}}(\alpha, \beta)\right)=\frac{n}{\beta} \mathbb{E} \log \left(1-e^{-\left(\alpha+\beta E_{1}\right)}\right)=n \cdot f(\alpha, \beta) .
$$

The quantity $f(\alpha, \beta)$ is free energy per state. We have $f(\alpha, \beta)>\frac{1}{\beta} \log \left(1-e^{-\alpha}\right)$.

As usual, we can define entropy $S_{n}(\alpha, \beta)$ as

$$
S_{n}(\alpha, \beta)=\beta\left(U_{n}(\alpha, \beta)-F_{n}(\alpha, \beta)\right) .
$$

Clearly, $S_{n}(\alpha, \beta)=n \cdot s(\alpha, \beta)$ where $s(\alpha, \beta)=\beta(u(\alpha, \beta)-f(\alpha, \beta))$ is entropy per state. We have

$$
s(\alpha, \beta)=\mathbb{E}\left(\frac{\beta E_{1}}{e^{\alpha+\beta E_{1}}-1}\right)-\mathbb{E} \log \left(1-e^{-\left(\alpha+\beta E_{1}\right)}\right) .
$$

Remark: Assume $n \uparrow \infty, \nu \uparrow \infty$ in such a way that particle density $\rho=\nu / n$ exists. Then, using the equation of state as defined by Eqs. (2.15-2.16), there is an expression of $\alpha$ in terms of $\rho$ and $\beta$, provided the system is in the diluted phase. The thermodynamical quantities per state $u(\alpha, \beta), f(\alpha, \beta)$ and $s(\alpha, \beta)$ can better be expressed in terms of $\rho$ and $\beta$. The functions $s(\alpha, \beta)$ and $\beta f(\alpha, \beta)$ are $\beta$-Legendre conjugates.

The existence of a limit for the thermodynamical quantities per state when the system becomes infinite is clear. See Beghian and Sheldon, [1], for similar considerations. $\diamond$

\section{Bose randomized occupancy and order statis- tics}

In practice, it is convenient to order the energy levels; by doing so, dependence is naturally introduced. Let then $\mathbf{E}_{(n)}:=\left(E_{(1), n}, . ., E_{(n), n}\right)$ be the order statistics of $\mathbf{E}_{n}$, with $E_{(1), n}<. .<E_{(n), n}$. As is well known that the joint probability density of this vector is

$$
f_{E_{(1), n}, . ., E_{(n), n}}\left(\epsilon_{1}, . ., \epsilon_{n}\right)=n ! \prod_{m=1}^{n} f_{1}\left(\epsilon_{m}\right) \cdot \mathbf{I}_{\left\{\epsilon_{1}<. .<\epsilon_{n}\right\}} .
$$


Thermodynamics under ranked energy levels is the same as the one under unranked ones. This is basically because for symmetric in the arguments functionals $\varphi\left(\mathbf{E}_{n}\right) \stackrel{\text { a.s. }}{=} \varphi\left(\mathbf{E}_{(n)}\right)$; conditionally on $\mathbf{E}_{n}$, thermodynamic functions involved separable and additive functionals of the form: $\varphi\left(\mathbf{E}_{n}\right)=\sum_{1}^{n} g\left(E_{m}\right)$ for different $g$. However, $\sum k_{m} E_{m} \stackrel{a . s .}{\neq} \sum k_{m} E_{(m), n}$ and this is why sampling under $\mathbf{E}_{n}$ does not reduce to sampling under $\mathbf{E}_{(n)}$.

For instance, given $\mathbf{E}_{(n)}$, the joint probability of occupancies $K_{(n), \alpha}(m)$ of $E_{(m), n} ; m=1, . ., n$ now reads

$$
\begin{aligned}
\mathbb{P}_{\mathbf{E}_{(n)}}\left(K_{(n), \alpha}(m)=k_{m} ; m=1, . ., n\right) & =\frac{1}{Z_{\mathbf{E}_{(n)}}(\alpha, \beta)} \prod_{m=1}^{n} e^{-\left(\alpha+\beta E_{(m), n}\right) k_{m}} \\
Z_{\mathbf{E}_{(n)}}(\alpha, \beta) & =\prod_{m=1}^{n} \frac{1}{1-e^{-\left(\alpha+\beta E_{(m), n}\right)}} .
\end{aligned}
$$

Exchangeability is lost; averaging over $\mathbf{E}_{(n)}$, the unconditional occupancy probability is

$$
\begin{gathered}
\mathbb{P}\left(K_{(n), \alpha}(m)=k_{m} ; m=1, . ., n\right):=\mathbb{E P}_{\mathbf{E}_{(n)}}\left(K_{(n), \alpha}(m)=k_{m} ; m=1, . ., n\right)= \\
\mathbb{E}\left[\prod_{m=1}^{n} e^{-\left(\alpha+\beta E_{(m), n}\right) k_{m}}\left(1-e^{-\left(\alpha+\beta E_{(m), n}\right)}\right)\right]= \\
n ! \int_{\epsilon_{1}<. .<\epsilon_{n}} \prod_{m=1}^{n} e^{-\left(\alpha+\beta \epsilon_{m}\right) k_{m}}\left(1-e^{-\left(\alpha+\beta \epsilon_{m}\right)}\right) f_{1}\left(\epsilon_{m}\right) d \epsilon_{m} .
\end{gathered}
$$

The occupation numbers of state $m$ with energy $E_{(m), n}$, namely $\mathbb{E}\left(K_{(n), \alpha}(m)\right)$, present some interest in practice. Here for instance, we get a randomized version of Planck statistics

$$
\mathbb{E}\left(K_{(n), \alpha}(m)\right)=\sum_{k_{m} \geq 1} \mathbb{P}\left(K_{(n), \alpha}(m) \geq k_{m}\right)=\mathbb{E}\left(\frac{1}{e^{\alpha+\beta E_{(m), n}}-1}\right)
$$

where, with $F_{1}$ the distribution function of $E_{1}\left(\bar{F}_{1}:=1-F_{1}\right), E_{(m), n}>0$ has density

$$
\frac{n !}{(m-1) !(n-m) !} f_{1}(\epsilon) F_{1}(\epsilon)^{m-1} \bar{F}_{1}(\epsilon)^{n-m} \text {. }
$$

Let us investigate the full ground-state occupancy distribution in more details.

Lemma 4 The grand canonical distribution of ground state occupancy is

(3.2) $\mathbb{P}\left(K_{(n), \alpha}(1) \geq k_{1}\right)=e^{-\alpha k_{1}}\left(1-\beta \int_{0}^{\infty}\left(1-F_{1}\left(\frac{x}{k_{1}}\right)\right)^{n} e^{-\beta x} d x\right)$. 
Proof: With $\mathbf{E}_{(n) \backslash 1}:=\left(E_{(2), n}, . ., E_{(n), n}\right), \phi_{(1)}(\beta):=\mathbb{E} e^{-\beta E_{(1), n}}$ and $k_{1} \in$ $\mathbb{N}_{0}$, the ground state $E_{(1), n}$ occupancy is

$$
\begin{aligned}
\mathbb{P}\left(K_{(n), \alpha}(1)=k_{1}\right) & =\mathbb{E}\left[\frac{e^{-\left(\alpha+\beta E_{(1), n}\right) k_{1}} Z_{\mathbf{E}_{(n) \backslash 1}}(\alpha, \beta)}{Z_{\mathbf{E}_{(n)}}(\alpha, \beta)}\right] \\
& =\mathbb{E}\left[e^{-\left(\alpha+\beta E_{(1), n}\right) k_{1}}\left(1-e^{-\left(\alpha+\beta E_{(1), n}\right)}\right)\right] \\
& =e^{-\alpha k_{1}}\left[\phi_{(1)}\left(\beta k_{1}\right)-e^{-\alpha} \phi_{(1)}\left(\beta\left(k_{1}+1\right)\right)\right] .
\end{aligned}
$$

With $\bar{F}_{1}\left(\epsilon_{1}\right):=\mathbb{P}\left(E_{1}>\epsilon_{1}\right), \bar{F}_{(1)}\left(\epsilon_{1}\right):=\mathbb{P}\left(E_{(1), n}>\epsilon_{1}\right)$, recalling $\bar{F}_{(1)}\left(\epsilon_{1}\right)=$ $\bar{F}_{1}\left(\epsilon_{1}\right)^{n}$, we get

$$
\phi_{(1)}(\beta):=\mathbb{E}\left[e^{-\beta E_{(1), n}}\right]=1-\beta \int_{0}^{\infty} \bar{F}_{1}\left(\epsilon_{1}\right)^{n} e^{-\beta \epsilon_{1}} d \epsilon_{1} .
$$

Therefore Eq. (3.2) holds.

This last formula allows to extract some limiting information on the ground state occupancy at critical point.

- A limit law at criticality from example $(i)$ : Assume $F_{1}(\epsilon) \sim_{\epsilon \downarrow 0} C \cdot \epsilon^{\theta}$ with $\theta>1$, as in Example (i). Approaching critical point, $\alpha \downarrow 0, \rho \uparrow \rho_{c}(\beta)=H_{\beta}(1)$ and so, with $k_{n, c}:=n \rho_{c}(\beta)$ the critical number of particles in the system

$$
\begin{gathered}
\mathbb{P}\left(K_{(n), 0}(1) \geq k_{n, c}^{1 / \theta} z\right)=1-\beta \int_{0}^{\infty}\left(1-F_{1}\left(\frac{x}{k_{n, c}^{1 / \theta} z}\right)\right)^{n} e^{-\beta x} d x \\
\sim_{n \uparrow \infty} 1-\beta \int_{0}^{\infty} e^{-n F_{1}\left(\frac{x}{k_{n, c}^{1 / \theta}}\right)} e^{-\beta x} d x \sim_{n \uparrow \infty} 1-\beta \int_{0}^{\infty} e^{-\frac{C}{\rho_{c}}\left(\frac{z}{x}\right)^{-\theta}} e^{-\beta x} d x=: \bar{G}(z)
\end{gathered}
$$

where it can be checked that $\bar{G}(z), z>0$, as a scale exponential mixture of the Fréchet distribution $e^{-\frac{C}{\rho_{c}} z^{-\theta}}$, is a complementary distribution function of some random variable $Z>0$. Thus, we obtain

Corollary 5 Fix $\beta>0$ and assume $F_{1}(\epsilon) \sim_{\epsilon \downarrow 0} C \cdot \epsilon^{\theta}$. Then, with $k_{n, c}:=$ $n \rho_{c}(\beta)$ the critical number of particles in the system at temperature $\beta^{-1}$, at critical point

$$
\frac{K_{(n), 0}(1)}{k_{n, c}^{1 / \theta}} \stackrel{d}{\rightarrow}_{n \uparrow \infty} Z .
$$

Ground state occupancy grows like $k_{n, c}^{1 / \theta}$ which is close to $n \rho_{c}$ only as $\theta$ approaches 1 from above. 
- Some remarks on the condensed phase: Fix $\beta>0$ and assume condensation takes place. In the super-critical condensed phase $\rho>\rho_{c}(\beta)$, there is no $\alpha$ such that Bose condition holds. Therefore, the grand canonical approach is useless to describe the condensed phase and, with $\kappa_{n}=\lfloor n \rho\rfloor$, one needs to use back the canonical ensemble description $K_{n, \kappa_{n}}(m), m=1, . ., n$ of the occupancy problem. We have the following result:

Proposition 6 Assume $\mathbb{E}\left(1 / E_{1}^{2}\right)<\infty$, so that $\mathbb{E}\left(1 / E_{1}\right)<\infty$ and condensation takes place. Let $\rho>\rho_{c}$ be the density of particles in the condensed phase. Under this assumption, almost surely

$$
\frac{1}{n} \sum_{m=2}^{n} \frac{e^{-\beta \widetilde{E}_{(m), n}}}{1-e^{-\beta \widetilde{E}_{(m), n}}} \underset{n \uparrow \infty}{\rightarrow} \rho_{c}=\rho_{c}(\beta)=\mathbb{E}\left[\frac{e^{-\beta E_{1}}}{1-e^{-\beta E_{1}}}\right]<\infty
$$

so that a proportion $n\left(\rho-\rho_{c}\right)$ of particles goes to ground state, while the residual $n \rho_{c}$ is scattered on all remaining energy levels.

Sketch of proof: First, the minimal value $E_{(1), n}$ from $\mathbf{E}_{n}$ goes to 0 almost surely. Under fairly general assumptions indeed (see Embrechts et al, [8]), the almost sure behavior of partial minima reads

$$
\lim \inf _{n \uparrow \infty} \frac{E_{(1), n}}{\epsilon_{n}}=1 \text { a.s. }
$$

where $\epsilon_{n} \downarrow 0$ is defined by $F_{1}\left(\epsilon_{n}\right)=n^{-1}$. We shall say $E_{(1), n} \sim \epsilon_{n}$.

Let now $g(x):=\left(e^{\beta x}-1\right)^{-1}$. If there is condensation (or if $\mathbb{E}\left(1 / E_{1}\right)<\infty$ ), then as $n \uparrow \infty$

$$
\frac{1}{n} g\left(E_{(1), n}\right) \rightarrow 0
$$

because, in the worst case $E_{(1), n} \sim n^{-1 / \theta}, \theta>1$ and then $\frac{1}{n} g\left(E_{(1), n}\right) \sim$ $n^{1 / \theta-1} \rightarrow 0$.

Recall now from Eq. (2.5) that when $k=\kappa_{n}$ is large, the joint law of occupancy numbers $K_{(n), \kappa_{n}}(m) ; m=2, . ., n$ for all states but ground state is (up to a constant going exponentially fast to 0 with $\kappa_{n} \uparrow \infty$ ) close to the one of a sequence of independent geometrically distributed random variables with success probabilities $e^{-\beta \widetilde{E}_{(m), n}}$, where $\widetilde{E}_{(m), n}:=E_{(m), n}-E_{(1), n}$ (see the proof of Proposition 1). Let us therefore investigate the large $n$ behavior of the sample mean number of particles

$$
\frac{1}{n} \sum_{m=2}^{n} \frac{e^{-\beta \widetilde{E}_{(m), n}}}{1-e^{-\beta \widetilde{E}_{(m), n}}}=: \frac{1}{n} \sum_{m=2}^{n} g\left(\widetilde{E}_{(m), n}\right)
$$

on all states but ground state. Recalling $E_{(1), n}$ goes to 0 almost surely, we have

$$
g\left(\widetilde{E}_{(m), n}\right) \sim \frac{1}{e^{\beta E_{(m), n}}\left(1-\beta E_{(1), n}\right)-1}
$$




$$
\sim \frac{1}{e^{\beta E_{(m), n}}-1}\left(1+\frac{\beta E_{(1), n}}{1-e^{-\beta E_{(m), n}}}\right) .
$$

Therefore,

$$
\frac{1}{n} \sum_{m=2}^{n} g\left(\widetilde{E}_{(m), n}\right) \sim \frac{1}{n} \sum_{m=2}^{n} g\left(E_{(m), n}\right)+\frac{\beta E_{(1), n}}{n} \sum_{m=2}^{n} \frac{e^{-\beta E_{(m), n}}}{\left(1-e^{\left.-\beta E_{(m), n}\right)^{2}} .\right.}
$$

Under the additional assumption $\mathbb{E}\left(1 / E_{1}^{2}\right)<\infty$, the corrective term goes to 0 when $n \uparrow \infty$ (with $\left.E_{(1), n} \downarrow 0\right)$. Indeed,

$$
\begin{aligned}
& \frac{\beta E_{(1), n}}{n} \sum_{m=2}^{n} \frac{e^{-\beta E_{(m), n}}}{\left(1-e^{-\beta E_{(m), n}}\right)^{2}}= \\
& \beta E_{(1), n}\left(\frac{1}{n} \sum_{m=1}^{n} \frac{e^{-\beta E_{(m), n}}}{\left(1-e^{-\beta E_{(m), n}}\right)^{2}}-\frac{1}{n} \frac{e^{-\beta E_{(1), n}}}{\left(1-e^{-\beta E_{(1), n}}\right)^{2}}\right)
\end{aligned}
$$

and, by strong law of large numbers,

$$
\frac{1}{n} \sum_{m=1}^{n} \frac{e^{-\beta E_{(m), n}}}{\left(1-e^{-\beta E_{(m), n}}\right)^{2}} \rightarrow \sigma^{2}=\mathbb{E}\left(\frac{e^{-\beta E_{1}}}{\left(1-e^{-\beta E_{1}}\right)^{2}}\right)
$$

which is a finite variance if $\mathbb{E}\left(1 / E_{1}^{2}\right)<\infty$. Next,

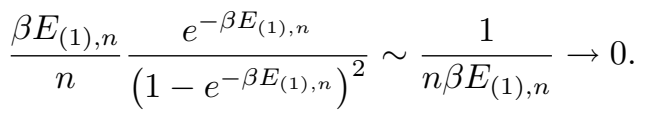

Therefore, $\frac{1}{n} \sum_{m=2}^{n} g\left(\widetilde{E}_{(m), n}\right) \sim \frac{1}{n} \sum_{m=1}^{n} g\left(E_{(m), n}\right)=\frac{1}{n} \sum_{m=1}^{n} g\left(E_{m}\right)$ and by strong law of large numbers

$$
\frac{1}{n} \sum_{m=2}^{n} g\left(\widetilde{E}_{(m), n}\right) \underset{n \uparrow \infty}{\rightarrow} \rho_{c}=\rho_{c}(\beta)=\mathbb{E}\left[g\left(E_{1}\right)\right]
$$

In a random scheme of allocating particles, it is said that a giant component appears if, as $k, n \rightarrow \infty$ while the ratio $\rho=k / n$ is fixed to $\rho>\rho_{c}$, the maximum $K_{(n), k}(1)$ of the random variables $K_{n, k}(m), m=1, . ., n$, with probability tending to 1 is of order $n$ and the second order statistic has a limit distribution with normalizing constant of order less than $n$. In the condensed phase, $K_{(n), k}(1)$ (the number of particles attached to ground state) is of order of magnitude $n\left(\rho-\rho_{c}\right)$. Let us investigate $K_{(n), k}(2)$ (the number of particles on state just above ground state) and check this from an example. 
First, $\mathbb{P}\left(E_{(1), n}>\epsilon_{1}\right)=\bar{F}\left(\epsilon_{1}\right)^{n}$ and, for $m=1, . ., n-1$ we have the Markov property:

$$
\begin{gathered}
\mathbb{P}\left(E_{(m+1), n}>\epsilon_{m+1} \mid E_{(m), n}=\epsilon_{m}, . ., E_{(1), n}=\epsilon_{1}\right)= \\
\mathbb{P}\left(E_{(m+1), n}>\epsilon_{m+1} \mid E_{(m), n}=\epsilon_{m}\right)=\left(\frac{\bar{F}_{1}\left(\epsilon_{m+1}\right)}{\bar{F}_{1}\left(\epsilon_{m}\right)}\right)^{n-m} ; \epsilon_{m+1}>\epsilon_{m}
\end{gathered}
$$

involving the cumulative probability distribution function of the minimum of $n-m$ iid observations drawn from $\bar{F}_{1}$ truncated at $\epsilon_{m}$. If $m=1$, with $\epsilon_{2}>\epsilon_{1}$

$$
\mathbb{P}\left(E_{(2), n}>\epsilon_{2} \mid E_{(1), n}=\epsilon_{1}\right)=\left(\frac{\bar{F}_{1}\left(\epsilon_{2}\right)}{\bar{F}_{1}\left(\epsilon_{1}\right)}\right)^{n-1}
$$

and so with $\epsilon>0$

$$
\begin{gathered}
\mathbb{P}\left(\widetilde{E}_{(2), n}>\epsilon \mid E_{(1), n}=\epsilon_{1}\right)=\left(\frac{\bar{F}_{1}\left(\epsilon_{1}+\epsilon\right)}{\bar{F}_{1}\left(\epsilon_{1}\right)}\right)^{n-1} \\
=\left(1-\frac{\bar{F}_{1}\left(\epsilon_{1}\right)-\bar{F}_{1}\left(\epsilon_{1}+\epsilon\right)}{\bar{F}_{1}\left(\epsilon_{1}\right)}\right)^{n-1}
\end{gathered}
$$

From this, when $n$ is large and if $F_{1}(\epsilon) \sim_{\epsilon \downarrow 0} C \cdot \epsilon^{\theta}, \theta>1$, we can indeed estimate $E_{(1), n}$ and $\widetilde{E}_{(2), n}$ to be both of order of magnitude $n^{-1 / \theta}$. Therefore, the average occupancy of state $E_{(2), n}$ is $g\left(\widetilde{E}_{(2), n}\right) \sim n^{1 / \theta}$ which is of order less than $n$.

\section{Sampling formulae}

- Sampling formula: Let $p \in\{1, . ., n\}$ and $1 \leq m_{1}<. .<m_{p} \leq n$ be an increasing subsequence of $\{1, . ., n\}$. Let $M_{1}, . ., M_{p}$ be the labels of occupied energy levels and $P_{n, \alpha}$ denote the number of such occupied states. With $k_{q} \in$ $\mathbb{N}:=\{1,2, .$.$\} , a realization of occupancies K_{(n), \alpha}\left(m_{q}\right)$, we clearly have

Proposition 7 (sampling formula)

$$
\begin{array}{r}
\mathbb{P}_{\mathbf{E}_{(n)}}\left(M_{q}=m_{q}, K_{(n), \alpha}\left(m_{q}\right)=k_{q} ; q=1, . ., p ; P_{n, \alpha}=p\right) \\
=\prod_{q=1}^{p}\left[e^{-\left(\alpha+\beta E_{\left(m_{q}\right), n}\right) k_{q}}\left(1-e^{-\left(\alpha+\beta E_{\left(m_{q}\right), n}\right)}\right)\right] \prod_{q \neq 1, \ldots, p}\left[1-e^{-\left(\alpha+\beta E_{\left(m_{q}\right), n}\right)}\right] .
\end{array}
$$

Averaging over $\mathbf{E}_{(n)}$ with joint law given by Eq. (3.1), gives the unconditional probability of the event $M_{q}=m_{q}, K_{(n), \alpha}\left(m_{q}\right)=k_{q} ; q=1, . ., p ; P_{n, \alpha}=p$. 
Summing next the above conditional probability over $k_{q} ; q=1, \ldots, p$, we get

$$
\begin{gathered}
\mathbb{P}_{\mathbf{E}_{(n)}}\left(M_{q}=m_{q} ; q=1, . ., p ; P_{n, \alpha}=p\right) \\
=\prod_{q \neq\{1, . ., p\}}\left[1-e^{-\left(\alpha+\beta E_{\left(m_{q}\right), n}\right)}\right] \times \\
\sum_{k_{1}, . ., k_{p} \geq 1} \prod_{q=1}^{p}\left[e^{-\left(\alpha+\beta E_{\left(m_{q}\right), n}\right) k_{q}}\left(1-e^{-\left(\alpha+\beta E_{\left(m_{q}\right), n}\right)}\right)\right] \\
=\prod_{q \neq\{1, . ., p\}}\left[1-e^{-\left(\alpha+\beta E_{\left(m_{q}\right), n}\right)}\right] \prod_{q=1}^{p} e^{-\left(\alpha+\beta E_{\left(m_{q}\right), n}\right)} .
\end{gathered}
$$

Averaging over $\mathbf{E}_{(n)}$, using the joint law of $\mathbf{E}_{(n)}$, we get the unconditional probability $\mathbb{P}\left(M_{q}=m_{q} ; q=1, . ., p ; P_{n, \alpha}=p\right)$ that only $p \leq n$ of the ordered energy levels with labels $m_{q}$ are occupied. In particular

$$
\mathbb{E}\left\{\prod_{q=2}^{n}\left[1-e^{-\left(\alpha+\beta E_{(q), n}\right)}\right] e^{-\left(\alpha+\beta E_{(1), n}\right)}\right\}
$$

is the unconditional probability that only ground state $E_{(1), n}$ is occupied.

Summing over the $\left(\begin{array}{l}n \\ p\end{array}\right)$ sequences $m_{1}<. .<m_{p}$, with $p \in\{0, . ., n\}$, we obtain a binomial distribution for the number of occupied energy states:

$$
\mathbb{P}\left(P_{n, \alpha}=p\right)=\left(\begin{array}{l}
n \\
p
\end{array}\right)\left[e^{-\alpha} \phi_{1}(\beta)\right]^{p}\left(1-e^{-\alpha} \phi_{1}(\beta)\right)^{n-p}
$$

Note that $\mathbb{P}\left(P_{n, \alpha}=0\right)=\left(1-e^{-\alpha} \phi_{1}(\beta)\right)^{n}$ which is the Gibbs probability that there is no particle in the system: $K_{n, \alpha}=0$.

Corollary 8 Fix $\beta>0$ and let $k_{n, c}:=n \rho_{c}(\beta)$ be the critical number of particles in the system. Then, as $n \uparrow \infty, \alpha \downarrow 0$, approaching critical point from the fluid phase

$$
\frac{P_{n, \alpha}}{n \rho} \sim \frac{P_{n, 0}}{k_{n, c}} \stackrel{d}{\rightarrow} \frac{\phi_{1}(\beta)}{\rho_{c}(\beta)}<1 .
$$

Proof: With $\lambda \geq 0$, the LST of $\frac{P_{n, 0}}{k_{n, c}}$ reads

$$
\mathbb{E}\left(e^{-\lambda \frac{P_{n, 0}}{k_{n, c}}}\right)=\left(1-\left(1-e^{-\frac{\lambda}{k_{n, c}}}\right) \phi_{1}(\beta)\right)^{n} \sim e^{-\frac{\lambda \phi_{1}(\beta)}{\rho_{c}(\beta)}} .
$$

From the state equation, $\rho_{c}(\beta)=H_{\beta}(1)=\sum_{i \geq 1} \phi_{1}(\beta i)>\phi_{1}(\beta)$. At critical point, the degenerate (non random) fraction $\frac{\phi_{1}(\bar{\beta})}{\rho_{c}(\beta)}$ of states is occupied. 
Remark (a non-degenerate low temperature $*$-limit at $\nu<\infty$ ): The above results suggest that the following "star" limit should also be worth considered: Assume $n \uparrow \infty, \beta \uparrow \infty$ (so that $\phi_{1}(\beta) \downarrow 0$ ) in such a way that $n \phi_{1}(\beta)=\gamma>0$.

Then

$$
P_{n, \alpha} \rightarrow_{*} P_{\alpha} \stackrel{d}{\sim} \text { Poisson }\left(\gamma e^{-\alpha}\right)
$$

Recalling

$$
\nu=n z h_{\beta}(z) \text { where } z h_{\beta}(z)=\sum_{i \geq 1} z^{i} \phi_{1}(\beta i),
$$

in case $r_{2}(\beta):=\phi_{1}(2 \beta) / \phi_{1}(\beta) \rightarrow_{\beta \uparrow \infty} 0$, to the dominant term in $\beta, z h_{\beta}(z) \sim_{*}$ $z \phi_{1}(\beta)$. Thus, $\nu \sim_{*} n z \phi_{1}(\beta) \sim_{*} \gamma z=\gamma e^{-\alpha}$. Thus, in this asymptotic, $P_{\alpha} \stackrel{d}{\sim}$ Poisson $(\nu)$ where $\nu$ is the finite expected number of particles in the system. Note that in such limiting situations, density $\rho=\nu / n \rightarrow_{*} 0$ : in the $*$-limit, both temperature and density vanish simultaneously. $\diamond$

- Asymptotics of the statistics of the number of energy states with prescribed amount of particles: The understanding of the number of occupied (and therefore also of unoccupied) states is part of the broader problem of the number of states with prescribed amount of particles. Let therefore

$$
A_{n, \alpha}(i):=\sum_{m=1}^{n} \mathbf{I}_{\left\{K_{n, \alpha}(m)=i\right\}} ; i \geq 0
$$

be the number of energy states with exactly $i$ particles. Clearly, $A_{n, \alpha}(0)=$ $n-P_{n, \alpha}$ is the number of free states and $\sum_{i>0} A_{n, \alpha}(i)=n$. The case $i=1$ $(i=2)$ corresponds to singleton (doubleton) states. Recall first

$$
\mathbb{P}\left(K_{n, \alpha}(m)=k_{m} ; m=1, . ., n\right)=\prod_{m=1}^{n} \mathbb{P}\left(\xi_{m, \alpha}=k_{m}\right)
$$

where $\left(\xi_{1, \alpha}, . ., \xi_{n, \alpha}\right)$ is an iid sequence with $\mathbb{P}\left(\xi_{1, \alpha} \geq k_{1}\right)=e^{-\alpha k_{1}} \phi_{1}\left(\beta k_{1}\right)$. From this, for all sequences $\left(a_{i} ; i \geq 0\right)$ satisfying the constraint $\sum_{i \geq 0} a_{i}=n$, we easily get:

$$
\mathbb{P}\left(A_{n, \alpha}(1)=a_{1}, . ., A_{n, \alpha}(i)=a_{i}, . .\right)=n ! \cdot \prod_{i=0}^{\sum_{l \geq 1} l a_{l}}\left\{\frac{\mathbb{P}\left(\xi_{1, \alpha}=i\right)^{a_{i}}}{a_{i} !}\right\}
$$

When $i \geq 1$, we have $A_{n, \alpha}(i)=\sum_{m=1}^{n} \mathbf{I}_{\left\{K_{n, \alpha}(m)=i\right\}}$, so that

$$
\begin{aligned}
& \mathbb{E}\left(A_{n, \alpha}(i)\right)=\sum_{m=1}^{n} \mathbb{P}\left(K_{n, \alpha}(m)=i\right)=\sum_{m=1}^{n} \mathbb{P}\left(\xi_{m, \alpha}=i\right) \\
& =n \mathbb{P}\left(\xi_{1, \alpha}=i\right)=n e^{-\alpha i}\left[\phi_{1}(\beta i)-e^{-\alpha} \phi_{1}(\beta(i+1))\right] .
\end{aligned}
$$


Thus, consistently,

$$
\begin{aligned}
\mathbb{E}\left(K_{n, \alpha}\right) & =\sum_{i \geq 1} i \mathbb{E}\left(A_{n, \alpha}(i)\right)=n \sum_{i \geq 1} i e^{-\alpha i}\left[\phi_{1}(\beta i)-e^{-\alpha} \phi_{1}(\beta(i+1))\right] \\
& =n \sum_{i \geq 1} e^{-\alpha i} \phi_{1}(\beta i)=n \mathbb{E}\left(\left(e^{\alpha+\beta E_{1}}-1\right)^{-1}\right) .
\end{aligned}
$$

Therefore, we obtain:

Proposition 9 (i) For all sequences $\left(a_{i} ; i \geq 0\right)$ satisfying $\sum_{i \geq 0} a_{i}=n$ :

(4.5) $\mathbb{P}\left(A_{n, \alpha}(1)=a_{1}, . ., A_{n, \alpha}(i)=a_{i}, ..\right)=n ! \cdot \prod_{i=0}^{\sum_{l \geq 1} l a_{l}}\left\{\frac{\mathbb{P}\left(\xi_{1, \alpha}=i\right)^{a_{i}}}{a_{i} !}\right\}$.

(ii) Assume $n \uparrow \infty, \beta \uparrow \infty$ in such a way that $n \phi_{1}(\beta):=\gamma>0$. In this weak $*$-limit, $\left(A_{n, \alpha}(i) ; i \geq 1\right)$ converges to a sequence of independent random elements with law given by $A_{\alpha}(i) \stackrel{d}{\sim}$ Poisson $\left(\lambda_{i}\right), i \geq 1$ where

$$
\lambda_{i}:=\gamma e^{-\alpha i}\left[r_{i}-e^{-\alpha} r_{i+1}\right]
$$

and

$$
r_{i}:=\lim _{\beta \uparrow \infty} r_{i}(\beta) \text { with } r_{i}(\beta):=\phi_{1}(\beta i) / \phi_{1}(\beta) .
$$

(iii) In the weak $*-$ limit, the number of visited states $P_{n, \alpha}$ converges to $P_{\alpha} \stackrel{d}{\sim}$ $\operatorname{Poisson}\left(\gamma e^{-\alpha}\right)$.

Proof: it remains to prove $(i i)$ and (iii).

Observing $\mathbb{P}\left(\xi_{1, \alpha}=0\right)=1-e^{-\alpha} \phi_{1}(\beta)$ and $a_{0}=n-\sum_{i \geq 1} a_{i}$, we have

$$
\begin{gathered}
\mathbb{P}\left(A_{n, \alpha}(1)=a_{1}, . ., A_{n, \alpha}(i)=a_{i}, . .\right)=\frac{n !}{\left(n-\sum_{i \geq 1} a_{i}\right) !}\left(1-e^{-\alpha} \phi_{1}(\beta)\right)^{n} \times \\
\prod_{i=1}^{\sum_{l \geq 1} l a_{l}}\left\{\frac{\left(\mathbb{P}\left(\xi_{1, \alpha}=i\right) /\left(1-e^{-\alpha} \phi_{1}(\beta)\right)\right)^{a_{i}}}{a_{i} !}\right\} \\
\sim_{*} n^{\sum_{i \geq 1} a_{i}} e^{-\gamma e^{-\alpha}} \prod_{i \geq 1}\left\{\frac{\phi_{1}(\beta)^{a_{i}}\left(e^{-\alpha i} r_{i}(\beta)-e^{-\alpha(i+1)} r_{i+1}(\beta)\right)^{a_{i}}}{a_{i} !}\right\} \\
\sim_{*} \prod_{i \geq 1} \frac{\lambda_{i}^{a_{i}} e^{-\lambda_{i}}}{a_{i} !} .
\end{gathered}
$$


In the last equivalence, we used $\gamma e^{-\alpha}=\sum_{i \geq 1} \lambda_{i}$. The law of $\left(A_{n, \alpha}(i) ; i \geq 1\right)$ therefore converges to the infinite product of Poisson factors

$$
\mathbb{P}^{*}\left(A_{\alpha}(1)=a_{1}, . ., A_{\alpha}(i)=a_{i}, . .\right)=\prod_{i \geq 1} \frac{\lambda_{i}^{a_{i}} e^{-\lambda_{i}}}{a_{i} !}
$$

with the announced finite-dimensional distributions in $(i i)$. The constants $\nu:=$ $\mathbb{E}^{*}\left(K_{\alpha}\right), \alpha>0$ and $\gamma>0$ are now related by

$$
\nu=\sum_{i \geq 1} i \lambda_{i}=\gamma \sum_{i \geq 1} e^{-\alpha i} r_{i}
$$

To prove (iii) again, observe $P_{\alpha}=\sum_{i \geq 1} A_{\alpha}(i)$ and use the Poisson decomposition.

Examples : - Assume $r_{2}(\beta):=\phi_{1}(2 \beta) / \phi_{1}(\beta) \rightarrow_{\beta \uparrow \infty} r_{2}=0$ as in Examples (ii) and (iii). Then for all $i \geq 2, r_{i}(\beta) \rightarrow r_{i}=0$ and so $\lambda_{i} \rightarrow 0(\beta \uparrow \infty)$. Thus, $A_{n, \alpha}(i) \neq 0$ with some positive probability only when $i=1$ (singletons) and $\mathbb{P}^{*}\left(A_{\alpha}(1)=a_{1}\right)=\frac{\lambda_{1}^{a_{1}} e^{-\lambda_{1}}}{a_{1} !}$ where $\lambda_{1}=\gamma e^{-\alpha}=\nu$. This asymptotic regime is the one of uniques. States whose occupancies cannot exceed 1 are currently obtained in a Fermi-Dirac context. We have no physical interpretation of this statement.

- Assume $r_{2}(\beta):=\phi_{1}(2 \beta) / \phi_{1}(\beta) \rightarrow \beta \uparrow \infty r_{2}=2^{-\theta}$ as in the regularly varying Example $(i)$. Then, $r_{i}(\beta) \sim_{\beta \uparrow \infty} i^{-\theta}$ and $\lambda_{i} \sim_{\beta \uparrow \infty} \gamma\left(1-e^{-\alpha}\right) e^{-\alpha i} i^{-\theta}$; configurations with $A_{n, \alpha}(i)>0$ exist for all $i \geq 1$. $\diamond$

\section{References}

[1] Beghian L. E.; Sheldon E. The random-variable canonical distribution. J. Phys. A: Math. Gen. 34 No 14, 2001, 2913-2921.

[2] Bingham, N. H.; Goldie, C. M.; Teugels, J. L. Regular variation. Encyclopedia of Mathematics and its Applications, 27. Cambridge University Press, Cambridge, 1987. 491 pp.

[3] Bose, S. N. Plancks gesetz und Lichtquantenhypothese. Zeit. fur Phys., 1924, 26, 178-181.

[4] Brézin, E. Physique Statistique. Cours édité par l' Ecole Polytechnique, 1993.

[5] Derrida, B. Random-energy model: an exactly solvable model of disordered systems. Phys. Rev. B (3) 24, 1981, no. 5, 2613-2626. 
[6] Derrida, B. The random energy model. Common trends in particle and condensed matter physics (Proc. Winter Adv. Study Inst., Les Houches, 1980).

[7] Derrida, B. Random-energy model: limit of a family of disordered models. Phys. Rev. Lett. 45, 1980, no. 2, 79-82.

[8] Embrechts, P.; Klüppelberg, C.; Mikosh, T. Modelling extremal events. Springer-Verlag, Applications of mathematics 33, 1997.

[9] Evans, M. R.; Majumdar, S. N.; Zia, R. K. P. Canonical analysis of condensation in factorised steady state. J. Stat. Phys., 2006, vol. 123, no 2, 357-390.

[10] Feller W., An introduction to probability theory and its applications. vol. 1 and 2. John Wiley and Sons, Second Edition, New York, 1971.

[11] Holst, L. On discrete spacings and the Bose-Einstein distribution. Contributions to Probability and Statistics. Essays in honour of Gunnar Blom. Ed. by Jan Lanke and Georg Lindgren, Lund, 169-177, 1985.

[12] Johnson, N. L.; Kotz, S. Urn models and their application. An approach to modern discrete probability theory. Wiley Series in Probability and Mathematical Statistics. John Wiley \& Sons, New York-London-Sydney, 1977. xiii+402 pp.

[13] Kolchin, V. F. Random mappings. Translated from the Russian. With a foreword by S. R. S. Varadhan. Translation Series in Mathematics and Engineering. Optimization Software, Inc., Publications Division, New York, 1986 .

[14] Lenoble, O.; Zagrebnov, V. Bose-Einstein condensation in the Luttinger-Sy model. 2006, math-ph/0604068. 